\title{
Análisis Exploratorio de las Prácticas Matemáticas de dos Estudiantes Mapuches en Colegios con y sin Educación Intercultural Bilingüe
}

\author{
Exploratory Analysis of the Mathematical Practices of two Mapuches
}

Students in Schools with and without Bilingual Intercultural Education

\author{
Sonia S. Salas* \\ Juan D. Godino ${ }^{* *}$ \\ Segundo Quintriqueo ${ }^{* * *}$
}

\begin{abstract}
Resumen
En este artículo presentamos resultados de un primer acercamiento a las prácticas matemáticas de dos estudiantes mapuches de escuelas públicas con y sin Educación Intercultural Bilingüe (EIB). Estos resultados son parte de una investigación en desarrollo sobre la articulación de las prácticas matemáticas propias de la cultura mapuche y la matemática escolar. Presentamos un análisis epistémico-cognitivo para identificar conflictos semióticos e identitarios en los estudiantes mapuches al iniciar la educación obligatoria en el campo de numeración. Los resultados del análisis de los sistemas de prácticas matemáticas, tanto institucionales como personales, muestran la necesidad de realizar investigaciones que aborden la articulación epistémica de la matemática mapuche y la matemática escolar, para favorecer el desarrollo cognitivo e identitario del estudiante mapuche.
\end{abstract}

Palabras clave: Prácticas matemáticas. Análisis epistémico-cognitivo. Conflicto semiótico. Desarrollo cognitivo e identitario.

\begin{abstract}
In this article, we present the results of a first approach to the mathematical practices of two Mapuche students of public schools with and without Bilingual Intercultural Education (EIB). These results are part of a research project in development on the linking of mathematical practices of Mapuche culture and school mathematics. We present an epistemic-cognitive analysis that seeks to show possible semiotic conflicts and identity in the Mapuche students at the start of learning numeration in compulsory education. The results of the analysis of mathematical practices show the need for research that addresses the epistemic articulation of the Mapuche and school mathematics, to promote the Mapuche student's cognitive and identity development.
\end{abstract}

Keywords: Mathematical practices. Epistemic-cognitive analysis. Semiotic conflict. Cognitive and identity development.

\footnotetext{
* Máster en Didáctica de la Matemática por la Universidad de Granada (UGR). Profesora de Matemáticas en la Corporación Municipal de Quilpué, Valparaíso, Chile. Dirección postal: Baquedano 960, 2421326. Quilpué, Valparaíso, Chile.E-mail: sbsalass@gmail.com

** Doctor en Matemáticas por la Universidad de Granada (UGR). Catedrático de Didáctica de la Matemática del Departamento de Didáctica de la Matemática de la Universidad de Granada (UGR), Granada, España. dirección postal: Facultad de Educación. Campus de Cartuja, 18071, Granada, España. E-mail: jgodino @ugr.es

Doctor en Educación por la Universidad de Extremadura (UEx). Profesor Asociado Permanente de la Universidad Católica de Temuco (UCT), Temuco, Cautín, Chile. Dirección postal: Rudecindo Ortega 02950, Temuco, Cautín, Chile.E-mail: squintri@uct.cl
} 


\section{Introducción}

Las investigaciones desde la sociología, la antropología y las ciencias de la educación han mostrado el complejo escenario de los procesos educativos en el actual modelo de la Educación Intercultural Bilingüe (EIB) para el contexto mapuche en Chile. Si bien el discurso de la EIB pretende valorizar las raíces culturales de la nación, en su aplicación no es más que otro modelo de aculturación del pueblo mapuche (QUINTRIQUEO; McGINITY, 2009). Rother (2005), en su investigación sobre el conflicto intercultural y la educación en Chile, en cuanto a los desafíos y problemas que presenta el actual modelo de la EIB, nos plantea que muchos de los profesores mapuches formados en el modelo educacional común para todo el país, sienten que fueron desarraigados de su cultura, en el marco de una escolarización monocultural, basada en una cultura dominante de corte occidental eurocéntrico (PINEAU, 2001; HARRIS, 2002). Esto fue concebido con el objetivo de materializar la homogeneización cultural y linguiística de las poblaciones en los territorios coloniales y al interior de los Estados nacionales. Como plantea Akkari (2009), este proyecto monocultural ha tenido las siguientes fuentes para su origen: 1) La sustitución de la educación religiosa por la alfabetización de masas; 2) El positivismo y la herencia filosófica de la Ilustración, orientadas a combatir la ignorancia de los hombres y conseguir su progreso y emancipación, con valores universales que se superponen a los marcos de vida de territorios particulares; 3 ) La consolidación del nacionalismo y la formación del espíritu patriótico de los futuros ciudadanos.

Las tres fuentes identificadas han configurado los lineamientos de la educación escolar que ha sido masificada en todo el mundo, instituyendo los parámetros para construir sociedades homogéneas. En este sentido, Mampaey y Zanoni (2015), al estudiar el caso de la educación en Flandes (Bélgica), identifican tres tipos de prácticas de educación monocultural. La primera es la práctica de la educación monolingüe, sostenida en una enseñanza en la lengua materna, lengua oficial de la sociedad mayoritaria, dedicada a fomentar exclusivamente las habilidades lingüísticas de la mayoría y prohibiendo a los estudiantes de grupos minoritarios comunicarse de manera informal en su propia lengua. La segunda práctica se relaciona con la exclusión de las minorías religiosas de los ambientes escolares, principalmente de las minorías no occidentales. La tercera práctica implica el uso de programas curriculares eurocéntricos, que tienen por objetivo socializar el conocimiento occidental. Cada una de estas prácticas tienen como finalidad, que los estudiantes de grupos minoritarios y minimizados adquieran las normas, valores y conocimientos de la sociedad 
mayoritaria, reproduciéndose el racismo epistémico institucional y perpetuando relaciones desiguales de poder (TUBINO, 2014).

En esa perspectiva, los profesores de origen mapuche y no mapuche han sido formados en un sistema educativo único nacional monocultural, por lo cual no poseen la formación para incorporar conocimientos educativos mapuches a la educación escolar (QUINTRIQUEO, 2010). Así, el desarraigo cultural de los profesores mapuches puede ser el resultado de la universalidad del currículo de matemáticas occidentalizado, imperante, que no atiende la diversidad cultural y el conocimiento propio de los estudiantes. Quilaqueo y Quintriqueo (2010, p. 353) nos plantean que, en contexto mapuche, la educación monocultural "ha sido el actual método de colonización y formación de los estados".

Como consecuencia de ello, se tiene una disminución o exclusión del conocimiento y cosmovisión de la cultura mapuche en la educación escolar (QUINTRIQUEO; MAHEUX, 2004). Esta aculturación escolar deja en evidencia los muchos casos de auto negación de la identidad mapuche de niños y jóvenes, desde muy pequeños, para ser aceptados o integrarse en otra comunidad de prácticas, la sociedad chilena de origen europeo occidental (QUINTRIQUEO; McGINITY, 2009). Esto ha significado, sin lugar a dudas, una pérdida del conocimiento ancestral de su cultura de origen en pro de la adopción de la cultura dominante occidentalizada. En este sentido, podemos decir que la enculturación matemática en su nivel formal (BISHOP, 1999) y los valores que transmite afecta a la consolidación identitaria ${ }^{1}$ del individuo en sociedad y la democratización del acceso al conocimiento (SKOVSMOSE, 2012).

En Salas, Godino y Oliveras (2015) hemos logrado evidenciar la existencia de sistemas de prácticas matemáticas propias del pueblo Mapuche, reconocidas por la institucionalidad chilena. La matemática viva (OLIVERAS, 2006) de la cultura de este pueblo, posee algunas características análogas al sistema de numeración decimal occidental. Esto nos motivó a realizar un primer acercamiento a las prácticas matemáticas de dos estudiantes mapuches de $2^{\circ}$ año de primaria, para la comprensión fenomenológica del problema a la luz de nuestro marco teórico.

Existe acuerdo en la comunidad científica sobre la importancia de los conocimientos previos, la contextualización de la enseñanza y aprendizaje y que las situaciones de aprendizajes sean significativas para los estudiantes. En Salas (2014) reportamos que los

\footnotetext{
${ }^{1}$ Imagen de uno mismo, legítima y positiva que fortalecen aspectos más profundos y más íntimos de la relación del hombre (individuo, persona) con el mundo y con los otros, pero también consigo mismo (QUINTRIQUEO; MCGINITY, 2009).
} 
programas de matemáticas y los libros de textos para la educación primaria en Chile no abordan el conocimiento previo del estudiante que viene con él a la escuela, desde su cultura de origen. Dichos recursos curriculares ponen énfasis en los conocimientos previos matemáticos, fijados por el currículo monocultural, que hacen referencia al nivel inmediatamente anterior.

Si los objetos matemáticos son símbolos de unidades culturales (D'AMORE; GODINO, 2007) que emergen de los sistemas de prácticas realizados por las personas ante determinadas situaciones-problemas (GODINO; BATANERO, 1994), entonces estamos frente a un sistema de prácticas matemáticas mapuches, mediadas por unas prácticas discursivas propias del konünpa kimün, epistemología del conocimiento mapuche (QUINTRIQUEO; TORRES, 2013).

En este artículo reportamos algunas cuestiones relativas a la distancia epistemológica entre el conocimiento matemático mapuche y el conocimiento matemático escolar presente en las prácticas matemáticas de los estudiantes mapuches en contextos distintos, con el fin de identificar, a priori, posibles conflictos semióticos y por supuesto identitarios en los primeros años de educación obligatoria.

Para ello, realizamos un análisis epistémico-cognitivo de la resolución de un problema matemático que busca explorar qué está pasando en las aulas donde se escolarizan los estudiantes mapuches. Este análisis nos permite plantear cuestiones abiertas a futuras investigaciones, desde un enfoque socio-crítico de la instrucción matemática y socioantropológico respecto del desarrollo identitario del estudiante mapuche.

Apoyados en las herramientas de análisis epistémico-cognitivo del Enfoque Ontosemiótico del Conocimiento y la Instrucción Matemáticos (EOS) (GODINO; BATANERO, 1994; GODINO, 2002; GODINO; BATANERO; FONT, 2007), identificamos posibles conflictos semióticos de significados en la dualidad personal/institucional. La visión etnomatemática nos permitió, asimismo, realizar el análisis sociocrítico de cómo la educación matemática puede afectar al desarrollo identitario del estudiante, si el conocimiento matemático de origen no se articula de manera adecuada con el conocimiento matemático escolar en los primeros años de escolarización.

\section{Fundamentación teórica metodológica}

\section{1 Área problemática}


La Ley General de Educación (MINISTERIO DE EDUCACIÓN, 2009a, Artículo 2² párrafo $1^{\circ}$ ), representa el actual marco institucional para la educación obligatoria en Chile y su principio orientador enmarca a la educación "en el respeto y valoración de los derechos humanos y de las libertades fundamentales, de la diversidad multicultural y de la paz y de nuestra identidad nacional".

La EIB entra en vigencia, gradualmente, a partir del año 2010 y, a partir del año 2014, de manera obligatoria, en los establecimientos con una matrícula de estudiantes de ascendencia indígena igual o superior al 20\% (MINISTERIO DE EDUCACIÓN, 2009b). Según la base de datos del Ministerio de Educación, en la actualidad, el $70 \%$ de los establecimientos educacionales del país poseen matrícula indígena (MINISTERIO DE EDUCACIÓN, 2005).

Los nuevos conflictos que surgen en la formación de los ciudadanos chilenos, a partir de la implementación de la EIB, han sido abordados por diversos investigadores (GAJARDO, 2012; QUINTRIQUEO; MAHEUX， 2004; QUILAQUEO; QUINTRIQUEO， 2010; TREVIÑO et al., 2013; ROTHER, 2005; QUINTRIQUEO; MCGINITY, 2009). Estos autores han puesto en discusión cuestiones como: la distancia epistémica entre el conocimiento mapuche y el conocimiento escolar, la elaboración de programas de estudios propios en las comunidades indígenas, tener un currículo intercultural a nivel país, sistematizar el conocimiento de las culturas originarias, como el conocimiento matemático, entre otros.

Los bajos rendimientos en matemáticas reflejan la discriminación social existente en relación a la distribución democrática del conocimiento matemático. Según Díaz y Druker (2007), la democratización del conocimiento en el aula escolar implica romper con la hegemonía del conocimiento escolar monocultural, para dar espacio y valor epistémico a las prácticas socioculturales fundadas, conocimiento propio de los niños de ascendencia indígena y no indígena. Proceso marcado, fuertemente, por la dimensión normativa institucional, que, en definitiva, condiciona el proceso de enseñanza y aprendizaje de la matemática en el aula en el actual modelo educativo monocultural.

Para este estudio nos planteamos las siguientes cuestiones:

1) ¿Comprenden los estudiantes mapuches sus prácticas matemáticas de origen y las pueden utilizar para resolver una tarea matemática en la escuela?

2) ¿Afecta la aculturación matemática en la escuela al desarrollo identitario del estudiante mapuche?

3) ¿Afecta la auto negación identitaria el aprendizaje de la matemática escolar?

Para responder a estas cuestiones hemos definido los siguientes objetivos: 
O1: Describir las prácticas matemáticas personales de dos estudiantes mapuches de $2^{\circ}$ año básico; uno en escuela con EIB y otro en escuela sin EIB.

O2: Analizar los significados personales e institucionales de los objetos matemáticos involucrados en la resolución de una tarea matemática.

O3: Identificar conflictos semióticos en el aprendizaje de la numeración en el contexto descrito.

\subsection{Aspectos teóricos}

En la comunidad científica se reconocen los aportes de connotados investigadores de la psicología de la educación, como Vigotsky, quien, ya en los años 50, nos hablaba del aprendizaje sociocultural del individuo. Han transcurridos varias décadas desde entonces para ver cómo el mundo, consecuencia de la globalización, es un lugar multicultural donde cotidianamente aprendemos de la cultura del otro. Como consecuencia, una educación intercultural debiera considerar las prácticas y las percepciones (D’AMBROSIO, 1999) de los estudiantes como una base en la cual afianzar el nuevo conocimiento, es decir, considerar su historia individual y su cultura de origen.

Por esta razón hemos decidido iniciar un trabajo exploratorio para evidenciar la necesaria articulación entre el conocimiento de origen del estudiante mapuche y la matemática escolar que estudian al iniciar la educación obligatoria. Atendiendo a las necesidades actuales de inserción del individuo en una sociedad globalizada y tecnificada, planteamos que el conocimiento de origen de un estudiante debe ser considerado un conocimiento previo en el cual afianzar los nuevos constructos, estableciendo una relación epistémica que articule, adecuadamente, estos distintos conocimientos matemáticos.

La resistencia de la matemática formal hacia la interdisciplinariedad (SKOVSMOSE, 1999) y la ausencia del componente sociocultural dentro de la disciplina de las matemáticas, no favorecen el desarrollo del pensamiento crítico e identitario de los estudiantes. Concordamos con Skovsmose (1999, p. 67), quien plantea

[...] es importante hacer crítica a la educación si se quiere que ésta no degenere en una manera de socializar efectivamente a los estudiantes en una sociedad tecnológica y, al mismo tiempo, aniquilar la posibilidad de que desarrollen una actitud crítica hacia, justamente, esa misma sociedad.

La etnomatemática está en referencia a grupos sociales y culturales diferenciados, a las concepciones e ideas, conceptos (saberes) y a diversas formas de prácticas socioculturales. En este contexto, "cada vez que los matemáticos tratan de abordar la etnomatemática, 
manifiestan diferencias, obstáculos o simplezas para analizarlos y terminan aplicando sólo su óptica profesional y disciplinaria" (ARAUJO, 2008, p. 71). En esa perspectiva, la etnomatemática requiere de profesores que se despojen de su visión monocultural, de sus prejuicios y del racismo, producto de su formación fundada en conocimientos científicos institucionalizados en una sociedad hegemónica en su relación histórica con los pueblos originarios (QUILAQUEO; QUINTRIQUEO, 2008). La finalidad es considerar el contexto sociocultural de los estudiantes, porque la matemática constituye una forma de pensamiento que se preserva en la identidad y se expresa en tres niveles de significación: 1) como visión de mundo, cosmovisión sobre el mundo físico, sobre el entorno natural y social; 2) cosmogonía, donde se explica los orígenes y poderes espirituales que permiten interpretar las concepciones sobre los elementos objetivos y subjetivos; y 3) la cosmología, que expresa los conceptos de orden, número, ritmo, lógica y percepciones del tiempo y del espacio (ARAUJO, 2008).

En nuestro estudio, la etnomatemática nos ha permitido mirar las cuestiones sociales y culturales en el conocimiento matemático del pueblo mapuche, mientras que la matemática crítica nos permite fundamentar los aspectos sociales y políticos. Visiones que permiten desarrollar una alternativa de educación matemática que exprese la conciencia social y la responsabilidad política (VITHAL; SKOVSMOSE, 1997) de la educación matemática en los primeros niveles de la educación obligatoria. Esta visión socio-crítica de la educación matemática promueve no desraizar al aprendiz de su cultura para pertenecer a otra mediante la adquisición de un nuevo conocimiento matemático, como lo ha reportado Rother (2005) en su estudio. Asimismo, la visión socio-crítica permite reflexionar sobre la exclusión que genera la educación matemática y sobre el acceso democrático al conocimiento matemático de manera inclusiva, valorando el conocimiento de la diversidad social y cultural de una nación y terminar con la política de obstáculos de aprendizaje (SKOVSMOSE, 2012). En otras palabras, disminuir las relaciones de poder que se establecen entre individuos, sociedades y culturas a través del conocimiento y desconocimiento matemático.

Skovsmose (2012) plantea una visión interesante de obstáculo de aprendizaje, desde la matemática crítica, que se entrecruza con la visión de la etnomatemática, en cuanto a la exclusión que provoca un modelo de currículo monocultural y universal. Plantea que la noción clásica de obstáculo podría ser un disfraz de exclusión. El no considerar la cultura de origen de los estudiantes al ingresar a la educación formal, puede ser asumido como una política de obstáculo de aprendizaje. Este planteamiento de Skovsmose ha dejado en 
evidencia que esta noción de obstáculo puede sepultar el porvenir ${ }^{2}$ del estudiante y arruinar el porvenir de un grupo de niños es un acto sociopolítico (SKOVSMOSE, 2012).

Vithal y Skovsmose (1997) describen cuatro facetas o campos de estudio de la etnomatemática: Historia de la matemática, Antropología cultural matemática, Matemáticas en la vida cotidiana y Relaciones entre etnomatemática y educación matemática. En Salas, Godino y Oliveras (2015) hemos reportado dos de estas facetas: la antropología cultural matemática, explorando la existencia y características de la aritmética mapuche y cómo es utilizada en la EIB; y las relaciones entre la etnomatemática mapuche y la matemática escolar, describiendo y analizando las regularidades e irregularidades morfosintácticas y morfomatemáticas de la numeración oral en español y mapunzugun (lengua mapuche). A partir de los cuatro puntos propuestos por Vithal y Skovsmose, y con el transcurso de nuestra investigación, nos dimos cuenta que es necesario incorporar un quinto campo de estudio: Articulación de la etnomatemática y la matemática escolar. Esto es, analizar las relaciones entre la etnomatemática y la matemática escolar, para articular estos conocimientos matemáticos de manera adecuada en un diseño didáctico que respete la lógica epistémica de cada conocimiento. Con ello se potencia la integración de los grupos socioculturalmente excluidos en una sociedad informacional en condiciones de igualdad.

En nuestro caso, este quinto campo de estudio nos conduce a la observación de las prácticas matemáticas de estudiantes mapuches. Centramos la atención sobre los componentes semióticos y epistemológicos puestos en juego en dichas prácticas matemáticas, esto es, sobre la naturaleza y tipo de los objetos cuyos significados se ponen en juego. Para Godino y Batanero (1994), las prácticas matemáticas pueden ser idiosincrásicas de una persona (prácticas personales) o compartidas en el seno de una institución (prácticas institucionales).

Estos autores consideran "práctica matemática a toda actuación o expresión (verbal, gráfica, simbólica, etc.) realizada por alguien para resolver problemas matemáticos, comunicar a otros la solución obtenida, validarla o generalizarla a otros contextos y problemas" (GODINO; BATANERO, 1994, p. 334). En este sentido, los autores conciben a las instituciones como comunidades de prácticas, e incluyen en ellas a las culturas, grupos étnicos y contextos socioculturales.

Nuestro estudio se ajusta a la visión de Godino y Batanero (1994), ya que aborda el estudio de los sistemas de prácticas en el seno de la cultura del pueblo mapuche y, por tanto, asumimos, al igual que el Enfoque Ontosemiótico del Conocimiento y la Instrucción

\footnotetext{
${ }^{2}$ Cómo percibe una persona las oportunidades que las situaciones sociales, políticas y culturales le proporcionan.
} 
Matemáticos (EOS), el postulado antropológico de la relatividad socio-epistémica de los sistemas de prácticas, de los objetos emergentes de las mismas y los significados.

Para el análisis de las prácticas matemáticas de los estudiantes mapuches, utilizamos las herramientas teóricas del EOS en sus facetas epistémica-cognitiva, por cuanto abordamos los significados institucionales y personales frente a tareas matemáticas. En la faceta epistémica abordamos los sistemas de prácticas institucionales, así como los objetos matemáticos (elementos lingüísticos, conceptos, procedimientos, propiedades y argumentos) y procesos puestos en juego al resolver una tarea matemática. En la faceta cognitiva analizamos los sistemas de prácticas personales puestos en juego en las respuestas de los estudiantes. Esto nos permite identificar conflictos semióticos que pueden explicar las dificultades a que se enfrentan los estudiantes mapuches al resolver un problema matemático en la cultura escolar y que pueden dificultar el acoplamiento de significados.

\subsection{Metodología}

Este estudio es cualitativo, exploratorio, descriptivo e interpretativo. El problema matemático presentado a los estudiantes lo hemos elaborado siguiendo la lógica didáctica propuesta por el currículo de matemática nacional en Chile, teniendo en cuenta las orientaciones curriculares para la EIB, los libros de texto del primer ciclo de primaria que se utilizan actualmente y la actividad sobre aprendizaje de la decena analizada en Godino et al. (2011).

La muestra, la componen dos casos-tipo (HERNÁNDEZ; FERNÁNDEZ; BAPTISTA, 2010), de estudiantes mapuches de $2^{\text {o }}$ año básico. Es una muestra guiada de fácil acceso. Uno de ellos es de una escuela con Educación Intercultural Bilingüe (EIB) de la región de La Araucanía, zona Sur de Chile, en la que se concentra la mayor cantidad de población Mapuche, 33,6\% de total de la población mapuche del país (INE, 2002). El segundo estudiante de una escuela sin EIB, de la región de Valparaíso, zona Centro de Chile, con una población mapuche de un 2,41\% de la población total Mapuche del país.

La tarea exploratoria propuesta a los estudiantes consistió en una ficha de trabajo en la que se solicita contar el número de huevos representados en una figura y en otra el número de manzanas (Figura 1). En un primer momento se solicita responder en mapunzugun (lengua mapuche) a las preguntas de recuento. En un segundo momento se solicita representar cuántas unidades y decenas de huevos hay en la figura; además, se pide completar en palabras y números la representación de la estructura aditiva de la cantidad total de huevos y de 
manzanas. Finalmente se presenta al estudiante la expresión con palabras (español y mapunzugun) de cuatro números y se solicita la expresión de la estructura aditiva en lenguaje simbólico matemático de éstos.

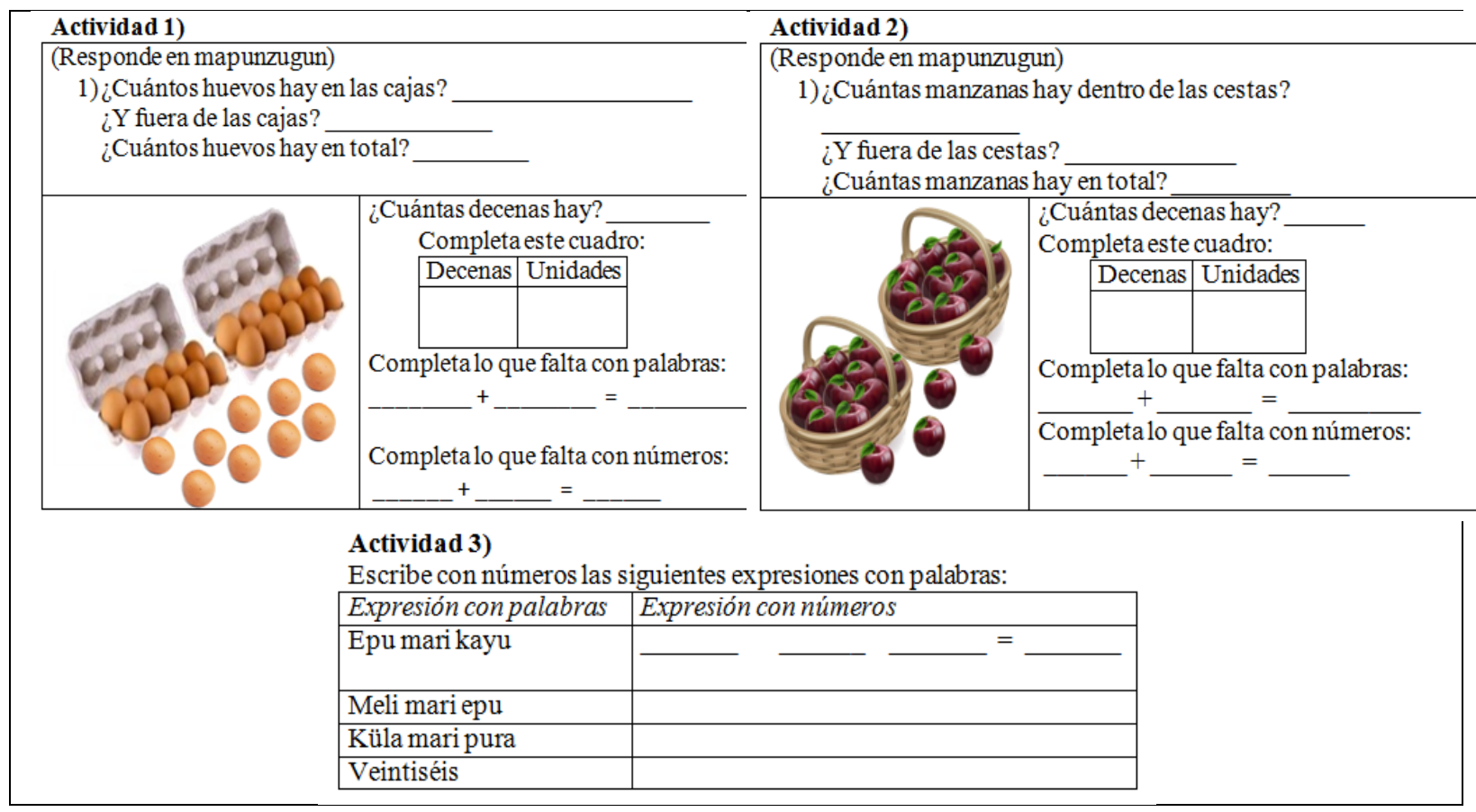

Figura 1 - Ficha de trabajo presentada a los estudiantes

Nuestro análisis epistémico-cognitivo de las prácticas matemáticas de estos estudiantes mapuches trata de aportar evidencias sobre las siguientes expectativas:

- Los estudiantes mapuches muestran su conocimiento del conteo en mapunzugun al solicitarles responder utilizando su lengua de origen.

- Los estudiantes mapuches evidencian el aprendizaje del valor posicional y lo demuestran en sus representaciones simbólico matemáticas o en sus prácticas discursivas en castellano o mapunzugun.

- A priori, planteamos que el estudiante que asiste a la escuela sin EIB, debe tener mayor conocimiento de la cultura matemática escolar. Sin embargo, esperamos, que aún pueda responder algunas cuestiones en mapunzugun.

- A priori, planteamos que el estudiante que asiste a la escuela con EIB, debe responder, con mayor facilidad, a las cuestiones solicitadas en mapunzugun. Sin embargo, puede ser que presente dificultad en la representación simbólica matemática.

Esperamos, que en ambos casos, los estudiantes sean capaces de identificar las unidades y decenas, representar las cantidades dadas en palabras de manera numérica de acuerdo al valor posicional y la yuxtaposición implícita en ellas (SALAS; GODINO; OLIVERAS, 2015). 


\section{Resultados y análisis}

\subsection{Sistemas de prácticas institucionales}

El Ministerio de Educación pone a disposición de las escuelas y liceos las Bases Curriculares para cada nivel de la educación obligatoria. Además, provee los Programas de Estudio de cada uno de los sectores de aprendizaje, a fin de facilitar y orientar la planeación de la enseñanza y el logro de los estándares fijados para cada nivel. Éstos sirven de guía didáctica para aquellos establecimientos educacionales que no posean sus propios programas de estudio. Los estándares de aprendizaje son indicadores de evaluación y permiten guiar la planeación de la enseñanza para el logro de los objetivos planteados en el currículo de matemática para cada nivel y que, al terminar cada ciclo de enseñanza, serán evaluados con la prueba nacional SIMCE (Sistema de Medición de la Calidad de la Enseñanza). En nuestro caso, hemos examinado el Programa de Estudio de matemáticas para $2^{\circ}$ año de la Educación Básica, centrando la atención en los objetivos de aprendizaje (OA), contenidos y competencias a desarrollar en este nivel sobre el conteo y valor posicional (MINISTERIO DE EDUCACIÓN, 2012).

Para la actual Educación Intercultural Bilingüe (EIB), el Ministerio de Educación (MINEDUC) y la Corporación Nacional de Desarrollo Indígena (CONADI) han elaborado en conjunto documentos curriculares para la contextualización de los contenidos fijados en los programas oficiales en los primeros niveles de Educación Básica de las siguientes asignaturas: Lenguaje y Comunicación, Matemática, Historia, Geografía y Ciencias Sociales, Educación Física y Salud, Artes Visuales y (MINISTERIO DE EDUCACIÓN, 2005).

El Cuadro 1 muestra los OA y los indicadores de logros establecidos por la institucionalidad para $2^{\circ}$ año básico que se relacionan con nuestro estudio. El primer OA implica el aprendizaje de leer los números cero, uno, dos,... en español y representarlos con objetos, en palabras y en lenguaje simbólico matemático. Implícitamente, el significado institucional (GODINO; BATANERO, 1994) pretende un aprendizaje asociado a la idea de cantidad, es decir, de manera transitiva (CID; GODINO; BATANERO, 2003). El segundo OA aborda el aprendizaje del valor posicional y se espera que los estudiantes sean capaces de representar y describir, de diferentes maneras, la formación de una cifra de dos dígitos. De manera implícita, el significado institucional pretendido es que los estudiantes sean capaces de representar la formación de una cifra demostrando la comprensión del valor posicional de 
un dígito asociado a la ubicación de éste y la comprensión de la estructura morfo-matemática de las palabras numéricas en español.

\begin{tabular}{|c|c|}
\hline Objetivo de Aprendizaje (OA) & Indicadores de Evaluación de logros \\
\hline $\begin{array}{l}\text { Leer números naturales del } 0 \text { al } 100 \text { y } \\
\text { representarlos en forma concreta, pictórica y } \\
\text { simbólica (OA 2) }\end{array}$ & $\begin{array}{l}\text { Escriben un número dado del } 0 \text { al 100, en cifras y en } \\
\text { Palabras }\end{array}$ \\
\hline $\begin{array}{l}\text { Identificar las unidades y decenas en números } \\
\text { del } 0 \text { al } 100 \text {, representando las cantidades de } \\
\text { acuerdo a su valor posicional, con material } \\
\text { concreto, pictórico y simbólico (OA 7) }\end{array}$ & $\begin{array}{l}\text { Identifican que el valor de un dígito depende de su } \\
\text { valor posicional dentro de un numeral. } \\
\text { Representan un número dado hasta } 50 \text {, en forma } \\
\text { concreta, pictórica y simbólica con el uso de material } \\
\text { multibase. } \\
\text { Ejemplo: } \\
\text { - } \square \square \square \text {.... } \\
\text { - } 30+4 \\
\text { - } 3 \text { decenas y } 4 \text { unidades } \\
\text { - } 34 \\
\text { Indican decenas y unidades en un número de dos } \\
\text { dígitos } \\
\text { Describen un número dado de dos dígitos, en el ámbito } \\
\text { hasta } 50 \text { de al menos dos formas. Ejemplo: } \\
34 \text { como } 3 \text { grupos de } 10 \text { con } 4 \text { unidades sobrantes ó } 34 \\
\text { como } 3 \text { decenas con } 4 \text { unidades, y también } 34 \text { unidades. }\end{array}$ \\
\hline
\end{tabular}

Cuadro 1 - Objetivos de Aprendizaje e Indicadores de logros Programa de Matemáticas $2^{\circ}$ año Básico

En las orientaciones didácticas en matemáticas para la EIB se plantean los OA e indicadores para el primer subciclo (NB1) que implica los niveles de $1^{\circ}$ y $2^{\circ}$ básico, desglosados en 4 semestres ( 2 años). En el Cuadro 2 incluimos los indicadores y objetivos de aprendizaje del primer semestre que tienen relación con nuestro tema de investigación.

\begin{tabular}{|l|l|}
\hline Objetivos de Aprendizaje (OA) & Indicadores \\
\hline $\begin{array}{l}\text { Manejan un procedimiento para contar hasta 30 } \\
\text { objetos y reconocen la importancia del conteo; } \\
\text { efectúan estimaciones y comparaciones de cantidades } \\
\text { en dicho ámbito numérico. }\end{array}$ & $\begin{array}{l}\text { Cuentan, en ambas lenguas, un conjunto de objetos } \\
\text { presentados en variadas formas y contextos. } \\
\text { Asocian el número obtenido al contar, en ambas } \\
\text { lenguas, con la cantidad de objetos de un conjunto } \\
\text { contado. } \\
\text { Dan ejemplos de situaciones en las que el conteo les } \\
\text { resulta necesario y útil }\end{array}$ \\
\hline $\begin{array}{l}\text { Reconocen el número que se forma a partir de una } \\
\text { suma de dos números dados y expresan un número } \\
\text { como la suma de otros dos, en el ámbito del 0 al 30. }\end{array}$ & $\begin{array}{l}\text { Identifican un número del ámbito del 0 al 30, formado } \\
\text { por la combinación de 10 ó 20, más un dígito. } \\
\text { Dan ejemplos, en forma oral y escrita, de números } \\
\text { de dos cifras hasta el 30, formados a partir de la suma } \\
\text { de 10, o de 20, más un dígito. } \\
\text { Dado un número menor que 30, lo descomponen de } \\
\text { diversas maneras, en sumas de otros dos. }\end{array}$ \\
\hline
\end{tabular}

Cuadro 2 - Objetivos de aprendizaje (OA) e indicadores de logros para NB1 en la EIB

Como podemos apreciar en el Cuadro 2, existen muchas más cuestiones implícitas que poseen un significado institucional que el profesor debe inferir para democratizar el conocimiento matemático escolar. Este significado promueve, desde el primer contacto con la escuela, el aprendizaje de la lectura y escritura de los números en dos lenguas (español y mapunzugun), asociados a la idea de cantidad. Se incluye también el aprendizaje del valor posicional en el lenguaje simbólico matemático y la representación descompuesta de una cifra 
en la suma del valor de sus dígitos. Sin embargo, no se aprecia la utilización del potencial educativo de la estructura morfo-matemática de la numeración oral en mapunzugun (SALAS; GODINO; OLIVERAS, 2015). Implícitamente, se espera que los estudiantes sean capaces de,

- comprender la estructura morfo-matemática de las palabras numéricas en español y mapunzugun (lengua mapuche);

- relacionar la escritura y verbalización de los números de dos dígitos en ambas lenguas;

- asociar las palabras numéricas en ambas lenguas al lenguaje simbólico matemático.

En este apartado podemos evidenciar la compleja tarea del profesor de matemáticas en el contexto mapuche en los primeros niveles de la educación obligatoria. El profesor debe comprender e inferir el significado institucional de referencia de la cultura escolar (CE) y de la cultura mapuche $(\mathrm{CM})$, para diseñar su planeación de enseñanza. Al diseñar la enseñanza debe responder a la demanda del currículo nacional de matemáticas, los estándares nacionales y las orientaciones curriculares para la EIB que insta al profesor a incorporar el lenguaje y contexto cultural mapuche. Una cuestión de interés para futuras investigaciones será explorar cómo puede el profesor articular estos aspectos en un diseño didáctico.

El conocimiento matemático del pueblo mapuche posee un sistema de conteo que lo podemos describir como un sistema naturalmente ordenado, oral, decimal no posicional. Éste posee algunas características análogas al sistema decimal posicional de la cultura occidental, como se aprecia en la tabla 1.

Tabla 1 - Sistema de Conteo Mapuche

\begin{tabular}{|c|c|c|c|c|c|}
\hline $\begin{array}{l}\text { Símbolo } \\
\text { Numérico }\end{array}$ & $\begin{array}{l}\text { En lengua } \\
\text { Mapunzugun }\end{array}$ & $\begin{array}{l}\text { Interpretación } \\
\text { aritmética }\end{array}$ & $\begin{array}{l}\text { Símbolo } \\
\text { Numérico }\end{array}$ & $\begin{array}{l}\text { En lengua } \\
\text { Mapunzugun }\end{array}$ & $\begin{array}{l}\text { Interpretación } \\
\text { aritmética }\end{array}$ \\
\hline 1 & Kiñe & 1 & 11 & Mari kiñe & $10+1$ \\
\hline 2 & Epu & $1+1$ & 12 & Mari epu & $10+2$ \\
\hline 3 & Küla & $2+1$ & 13 & Mari küla & $10+3$ \\
\hline 4 & Meli & $3+1$ & 14 & Mari meli & $10+4$ \\
\hline 5 & Kechu & $4+1$ & 15 & Mari kechu & $10+5$ \\
\hline 6 & Kayu & $5+1$ & 16 & Mari kayu & $10+6$ \\
\hline 7 & Regle & $6+1$ & 17 & Mari regle & $10+7$ \\
\hline 8 & Pura & $7+1$ & 18 & Mari pura & $10+8$ \\
\hline 9 & Aylla & $8+1$ & 19 & Mari aylla & $10+9$ \\
\hline 10 & Mari & $9+1$ & 20 & Epu mari & $2(10)$ \\
\hline 100 & Pataka & $10(10)$ & 1000 & Waragka & $10(100)$ \\
\hline 200 & Epu pataka & $2(100)$ & 2000 & Epu waragka & $2(1000)$ \\
\hline 300 & Kila pataka & $3(100)$ & 5000 & Kechu waragka & $5(1000)$ \\
\hline 400 & Meli pataka & $4(100)$ & 9999 & $\begin{array}{l}\text { Aylla waragka aylla } \\
\text { pataka aylla mari aylla }\end{array}$ & $\begin{array}{l}9(1000)+9(100)+ \\
9(10)+9\end{array}$ \\
\hline 500 & Kechu pataka & $5(100)$ & 10000 & mari waragka & $10(1000)$ \\
\hline
\end{tabular}

GODINO et al., (2011, p. 13) plantean que "los números, la aritmética, es la respuesta social al problema de comunicar el tamaño o numerosidad de los conjuntos, ordenar una colección y de analizar procesos iterativos-recurrentes". Así, el pueblo mapuche comenzó su 
propia forma de dar respuesta a este problema en su vida cotidiana, es decir, en un contexto informal con sus propias prácticas informales. En estas prácticas de conteo mapuches existe un sistema de objetos organizados con un primer elemento, un siguiente..., que les ha permitido dar respuesta a la pregunta ¿cuántos hay?, determinando el tamaño de una colección de objetos (cardinal). La habilidad de contar, en español o mapunzugun, está precedida de una coordinación, al mismo tiempo, entre los elementos a contar con alguna técnica de recuento, y la emisión de la palabra cuantificadora en el orden establecido por ellos como cultura. Por tanto, y como plantea Cid, Godino y Batanero (2003), las técnicas de contar para obtener los cardinales, en el caso de la cultura mapuche, ponen de manifiesto los principios necesarios para contar correctamente:

- Principio de orden estable. Las palabras numéricas kiñe, epu, küla... deben recitarse siempre en el mismo orden, sin saltarse ninguna.

- Principio de la correspondencia uno a uno. A cada elemento del conjunto sometido a recuento se le debe asignar una palabra numérica distinta y sólo una.

- Principio de irrelevancia del orden. El orden en que se cuentan los elementos del conjunto es irrelevante para obtener el cardinal del conjunto.

- Principio cardinal. La palabra adjudicada al último elemento contado del conjunto representa, no sólo el ordinal de ese elemento, sino también el cardinal del conjunto.

Al observar y conocer estos elementos de la matemática mapuche, podemos identificar en el proceso de contar la puesta en correspondencia biyectiva de cada elemento de un conjunto con una, y sólo una, palabra numérica verbalizada de acuerdo al orden establecido en cultura correspondiente, y respetando sus principios de conteo, que, análogamente, son similares a los de la cultura matemática occidental.

Estas características de los números mapuches y su estructura nos han motivado a indagar, primero, si la aculturación temprana del estudiante mapuche ha borrado ese conocimiento matemático de origen o aún se mantiene en el estudiante de segundo básico. En este sentido toma relevancia la exploración y comparación de contextos distintos en que aprenden matemáticas los estudiantes mapuches; esto nos permite observar las diferencias en las prácticas matemáticas y los significados personales de los estudiantes.

Los significados institucionales de referencia, en este estudio, son aquellas prácticas esperadas y definidas por los indicadores del currículo de matemáticas y las orientaciones didácticas para contexto indígena (ver cuadros 1 y 2) para este nivel educativo, y se compone de las siguientes acciones:

- Leer y comprender la tarea. 
- Contar el número de huevos representados en el dibujo.

- Escribir el resultado de los recuentos de formas diferentes:

- Lenguaje natural en mapunzugun o español.

- Como suma del cardinal de dos sub-colecciones (huevos dentro y fuera de las cajas).

- Lenguaje simbólico matemático del cardinal (número 29).

- Identificar la decena y las unidades del resultado y escribirlas en una tabla.

- Escribir expresiones numéricas con palabras en lenguaje simbólico matemático de manera aditiva.

\subsection{Sistemas semióticos personales}

La práctica esperada del alumno será la lectura y comprensión de la tarea y su resolución. El análisis a priori realizado permite formular algunas hipótesis relativas a los comportamientos de los estudiantes al resolver las tareas (aciertos, errores y diferentes maneras de abordarlas). La figura 2 muestra las respuestas de los estudiantes a dos de las tareas planteadas.

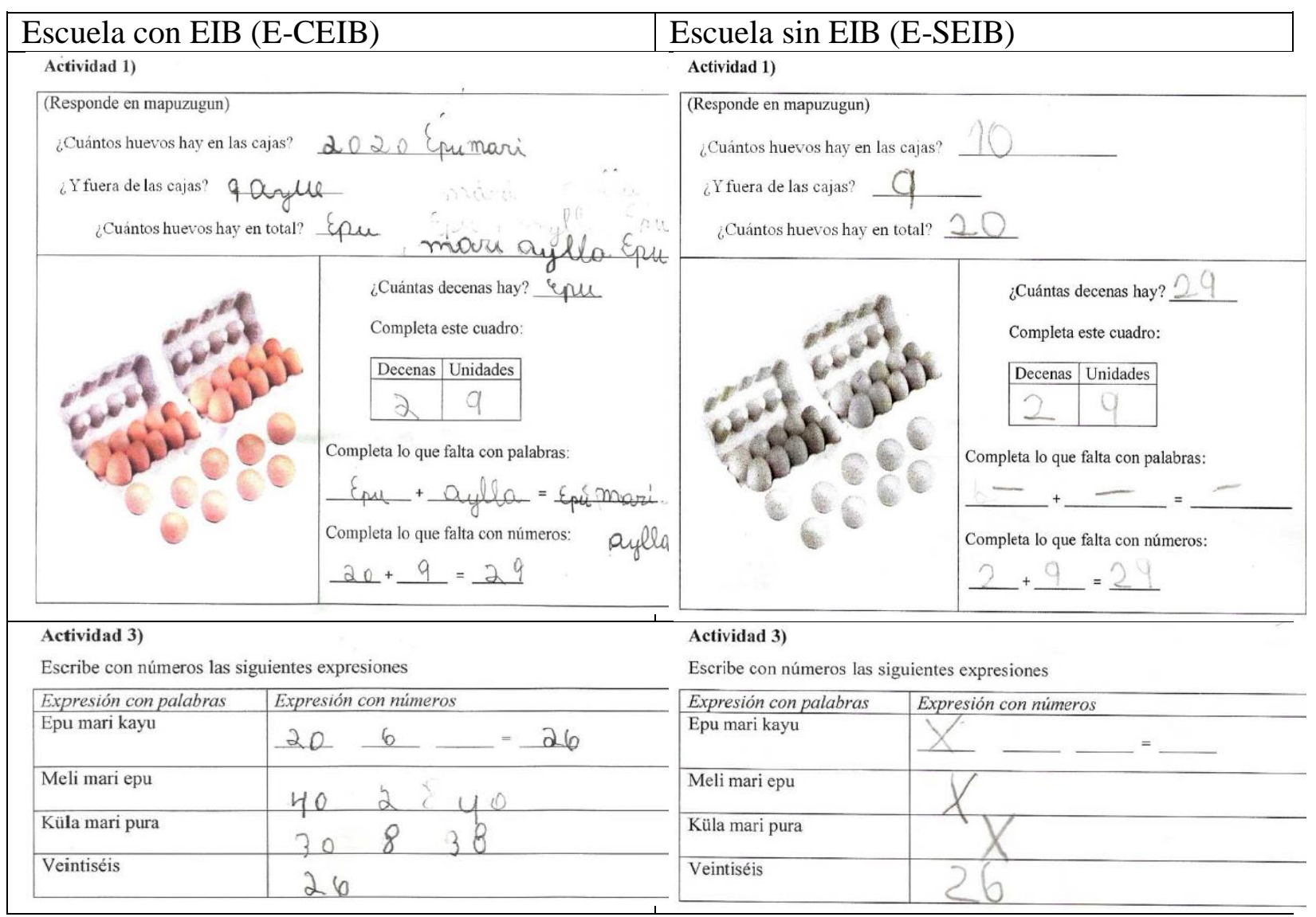

Figura 2- Respuestas de los estudiantes 
En ambos casos se pide contar la cantidad de huevos representados, solicitando escribir en mapunzugun. A continuación, se solicita encontrar el cardinal, expresado en decenas y unidades, y finalmente asociar las prácticas en lenguaje natural a la formación numérica de una cifra, como se aprecia en la figura 2. No se consideró entregar un ejemplo de resolución, dado que, según la revisión realizada del currículo y los libros de textos, se viene trabajando de esta forma desde el primer año. En nuestra aplicación, modificamos las colecciones a contar, huevos y manzanas, e introdujimos el mapunzugun, lengua originaria del estudiante mapuche.

En este acercamiento a las prácticas matemáticas de estos estudiantes mapuches, no hemos podido observar la forma en que los niños cuentan, ya que no se aprecia ninguna técnica auxiliar de recuento en la hoja de resolución. El estudiante con EIB (E-CEIB), aparentemente cuenta correctamente, identifica las decenas y las unidades, y las representa numéricamente de manera correcta. Sin embargo, el conocimiento de origen (escritura en mapunzugun) no se aprecia claramente, debido a que escribe algunas palabras correctamente, otras lo hace con errores y en otras no las escribe. Se aprecia que no comprende la formación de la cifra en mapunzugun (epu mari es 2 veces 10), ya que el estudiante lo escribe como 2020. Si bien muestra la representación como suma de dos subconjuntos de elementos, en otras instancias en que debía aplicar la misma representación, no lo hace. Cabe destacar que la mayor dificultad del E-CEIB se presenta cuando se involucran prácticas en lenguaje natural, lo que muestra un débil acoplamiento de los significados de las palabras numéricas en ambos lenguajes y su representación simbólica matemática de acuerdo a su estructura aditiva. El estudiante $\sin$ EIB (E-SEIB) no logra el recuento y sólo identifica el cardinal de las unidades. El significado personal de este estudiante no distingue entre el valor relativo y absoluto de las cifras ( 2 decena son 20 unidades). No ha construido el significado esperado para las unidades de primer orden ni las de segundo orden (decenas). No muestra conocimiento de la representación como suma de dos subconjuntos de elementos. En cuanto a su conocimiento de origen, no muestra evidencia alguna que recuerde los números en mapunzugun u otro conocimiento en relación al tema abordado.

Esta inconsistencia no se puede valorar únicamente en términos dicotómicos, el alumno sabe-no-sabe. Por ello, con este trabajo mostramos la complejidad semiótica que supone asociar las prácticas discursivas y los significados institucionales de la cultura escolar, la numeración oral en castellano y mapunzugun, y su asociación a la escritura simbólica matemática occidental. 
El caso que hemos descrito reafirma la complejidad del aprendizaje de la decena en los primeros años de escolaridad, resaltada por Godino et al. (2011) mediante el análisis de las prácticas, objetos y procesos involucrados en la resolución de una tarea similar a la que en este trabajo hemos utilizado. En nuestro caso, hemos revelado que esa complejidad ontosemiótica (reconocimiento de objetos intervinientes en las prácticas matemáticas requeridas y la puesta en funcionamiento de los procesos de semiosis implicados) es mayor en el caso del estudiante que aporta un conocimiento base sobre numeración proveniente de su cultura de origen. Se requiere establecer nuevas funciones semióticas entre los términos numéricos de la lengua mapunzugun, el español, los símbolos indo-arábigos y las reglas de los respectivos sistemas de numeración. Queda abierta, no obstante, la cuestión de si es posible sacar alguna ventaja desde el punto de vista del aprendizaje de la numeración decimal indoarábiga por parte del niño que conoce la lengua mapuche y los procesos de conteo en dicha cultura. En todo caso, será necesario tener en cuenta dicho conocimiento en la enseñanza de la aritmética en la escuela para lograr una educación inclusiva que respete la identidad cultural del niño mapuche.

\section{Reflexiones finales}

En las respuestas de los estudiantes a la tarea se aprecia que el estudiante de escuela con EIB tiene mayor comprensión de lo que se le solicita responder. En cambio, en la actuación del estudiante de escuela sin EIB no se aprecia que comprenda el problema y por ello presenta más errores en sus respuestas. En ocasiones, las respuestas muestran una comprensión de los signos (S) que se les presenta, sin embargo no se aprecia la comprensión del conjunto de reglas (R) y menos aún la relación entre el signo y su significado (M) (ERNEST, 2006). Ambos estudiantes muestran en sus prácticas el conflicto semiótico que representa la comprensión de la morfosintaxis de las palabras numéricas en español y mapunzugun, y su estructura morfo-matemática (SALAS; GODINO; OLIVERAS, 2015).

Para concluir, formulamos algunas cuestiones abiertas que requieren investigación sistemática, tales como:

- Reconstruir el conocimiento matemático del estudiante mapuche que viene con él a la escuela, para proponer diseños didácticos que articulen este conocimiento con la matemática escolar.

- Elaborar, implementar y evaluar diseños didácticos que articulen el conocimiento matemático mapuche con la matemática escolar de manera adecuada, en igualdad epistémica, 
para favorecer el desarrollo identitario del estudiante mapuche e identificar ventajas o dificultades de aprendizaje en el proceso.

- Establecer niveles de complejidad semiótica en el aprendizaje de las palabras numéricas (español y mapunzugun), a partir de las prácticas discursivas en los primeros años de educación obligatoria, en contexto mapuche.

- Dado que la dimensión normativa institucional condiciona los procesos de enseñanza y aprendizaje de la matemática escolar, se requiere investigar de qué manera esta dimensión se puede flexibilizar para promover el acercamiento epistémico del conocimiento matemático mapuche y la matemática escolar.

Estas cuestiones son un llamado de atención a distintos actores involucrados en los procesos de enseñanza y aprendizaje de la matemática. No sólo a los profesores de matemáticas, ya que la mayoría de éstos no han recibido formación sobre el conocimiento matemático del pueblo mapuche (QUILAQUEO; QUINTRIQUEO, 2010). Entre los actores involucrados tenemos los expertos en currículo, quienes diseñan los programas de estudio monoculturales y no asumen la condición de país multicultural. Las editoriales de libros de textos, que al parecer desconocen la existencia de escuelas indígenas y cuyos contenidos debieran contextualizarse a la vida cotidiana de estos estudiantes. A los formadores de profesores de matemáticas, quienes deben formar profesionales reflexivos y no meros consumidores de matemática occidental, los que luego replican su formación en el aula.

Es necesario reflexionar sobre estos y otros aspectos del proceso de enseñanza de la matemática escolar para comprender los aspectos sociopolíticos de la educación matemática que generan exclusión junto al discurso de inclusión (D’AMBROSIO, 2000) y podrían establecer anticipadamente una política de obstáculos de aprendizaje (SKOVSMOSE, 2012). Lograr la democratización del conocimiento matemático implica, necesariamente, una mayor equidad en los procesos de enseñanza que flexibilice la universalidad del currículo de matemática occidentalizado y posibilite mayores espacios de atención a la diversidad de conocimientos que confluyen en el aula de matemáticas.

Reconocimiento: Trabajo realizado parcialmente en el marco del proyecto de investigación, EDU2012-31869, Ministerio de Economía y Competitividad (MINECO, España), el Programa de Capital Humano Avanzado de la Comisión Nacional Científica y Tecnológica, CONICYT BECAS CHILE 72150172 y la Corporación Municipal de Quilpué, Chile. 


\section{Referencias}

AKKARI, A. Introduction aux approches interculturelles en éducation. Université de Genève, Faculté de psychologie et des sciences de l'éducation. 2009. Disponible en: <http://archiveouverte.unige.ch/unige:21763>. Acceso en: 10 abr. 2015.

ARAUJO, A. Una propuesta metodológica en Etnomatemáticas. Revista U. D. C. A Actualidad y Divulgación Científica, Bogotá, v. 11, n. 1, p. 67-76, 2008.

BISHOP, A. J. Enculturación matemática: la educación matemática desde una perspectiva cultural. Barcelona: Paidós, 1999.

CID, E.; GODINO, J. D.; BATANERO, C. Sistemas numéricos y su didáctica para maestros. Universidad de Granada. 2003. Disponible en: <http://www.ugr.es/local/jgodino/>. Acceso en: 24 oct. 2013.

D'AMBROSIO, U. La transferencia del conocimiento matemático a las colonias: factores sociales, políticos y culturales. Llull, Zaragoza, v. 22, n. 44, p. 347-380, 1999

D'AMBROSIO, U. Las dimensiones políticas y educacionales de la etnomatemática. Números, Canarias, n. 43, p. 439-444, 2000.

D'AMORE, B.; GODINO, J. D. El enfoque ontosemiótico como un desarrollo de la teoría antropológica en didáctica de la matemática. Revista latinoamericana de investigación en matemática educativa, México, v. 10, n. 2, p. 191-218, 2007.

DÍAZ, T.; DRUKER, S. La democratización del espacio escolar: una construcción en y para la diversidad. Estudios Pedagógicos, Santiago, v. 33, n. 1, p. 63-77, 2007.

ERNEST, P. A semiotic perspective of mathematical activity: The case of number. Educational Studies in Mathematics, Holanda, v. 61, n. 1-2, p. 67-101, 2006.

GAJARDO, A. Caracterización del rendimiento escolar de niños y niñas mapuches: contextualizando la primera infancia.2012. 361p. Tesis (Doctorado en Interculturalidad)- Facultad de Educación y Trabajo Social. Universidad de Valladolid, Valladolid, España, 2012.

GODINO, J. D. Un enfoque ontológico y semiótico de la cognición matemática. Recherches en didactique des Mathématiques, Grenoble, v. 22, n. (2/3), p. 237-284,2002

GODINO, J. D.; BATANERO, C. Significado institucional y personal de los objetos matemáticos. Recherches en didactique des Mathématiques, Grenoble, v. 14, n. 3, p. 325-355, 1994

GODINO, J. D.; BATANERO, C.; FONT, V. The onto-semiotic approach to research in mathematics education. ZDM. The International Journal on Mathematics Education, Berlin, v. 39, n. 1-2, p. 127-135, 2007.

GODINO, J. D.; FONT, V.; WILHELMI, M. R.; LURDUY, O. Why is the learning of elementary arithmetic concepts difficult? Semiotic tools for understanding the nature of mathematical objects. Educational Studies in Mathematics, Holanda, v. 77, n. 2-3, p. 247-265, 2011.

HARRIS, H. Coyote goes to school: The paradox of Indigenous higher education. Canadian journal of native education, Canada, v. 26, n. 2, p. 187-196, 2002.

HERNÁNDEZ, R.; FERNÁNDEZ, C.; BAPTISTA, M. Metodología de la investigación. 5. ed. México: Mc Graw Hill. 2010. 
InSTITUTO NACIONAL DE eSTADÍSTICA - INE. Censo de Población y vivienda. Chile, 2002. Disponible en: <http://www.ine.cl/canales/chile_estadistico/censos/censo_poblacion_vivienda.php>. Acceso en:16 jul. 2014.

MAMPAEY, J. y ZANONI, P. Reproducing monocultural education: ethnic majority staff's discursive constructions of monocultural school practices. British Journal of Sociology of Education. 2015. Disponible en: <http://www.tandfonline.com/doi/abs/10.1080/01425692.2014.1001059>. Acceso en: 23 abr. 2015.

MINISTERIO DE EDUCACIÓN. Orientaciones para la contextualización de Planes y Programas para la Educación Intercultural Bilingüe. Santiago: Chile, 2005.

MINISTERIO DE EDUCACIÓN. Ley General de Educación N² 20.370. Santiago: Chile, 2009a.

MINISTERIO DE EDUCACIÓN. Marco Curricular Lengua Indígena para la Educación Básica. Santiago: Chile, 2009b.

MINISTERIO DE EDUCACIÓN. Programa de Estudio de Matemáticas para $2^{\circ}$ año de educación básica. Santiago: Chile, 2012.

OLIVERAS, M. L. Etnomatemáticas: de la multiculturalidad al mestizaje. In: GOÑI, J.; ALBERTÍ, M.; BURGOS, S.; DÍAZ, R.; DOMINGUEZ, M.; FIORITI, G.; GORGORIÓ, N.; NUNES, CH.; OLIVERAS, M.L.; PLANAS, N.; PRAT, M.; ROJAS, F.J.; SANTESTEBAN, M.; VILELLA, X. (Coord.). Matemática e Interculturalidad. Barcelona: GRAÓ. 2006. p. 117-149.

PINEAU, P. ¿Por qué triunfó la escuela? O la modernidad dijo: esto es educación y la escuela respondió "yo me ocupo". In: PINEAU, P. (Coord.). La escuela como máquina de educar. Tres escritos sobre un proyecto de la modernidad. Buenos Aires: Paidós. 2001, p. 306-331.

QUILAQUEO, D.; QUINTRIQUEO, S. Formación docente en educación intercultural para contexto mapuche en Chile. Revista Cuadernos Interculturales, Valparaíso, v. 6, n. 10, p. 91-110. 2008.

QUILAQUEO, D.; QUINTRIQUEO, S. Saberes educativos mapuches: un análisis desde la perspectiva de los kimches. Polis, Santiago, v. 9, n. 26, p. 337-360. 2010.

QUINTRIQUEO, S. Implicancias de un modelo curricular monocultural en contexto mapuche. Santiago: Lom Ediciones, 2010.

QUINTRIQUEO, S.; MAHEUX, G. Exploración del conocimiento sobre la relación de parentesco como contenido educativo para un currículum escolar intercultural en comunidades mapuche. Revista de Psicología, Santiago, v. 13, n. 1, p. 73-91. 2004.

QUINTRIQUEO, S.; MCGINITY, M. Implicancias de un modelo curricular monocultural en la construcción de la identidad sociocultural de alumnos/as mapuches de la IX región de La Araucanía, Chile. Estudios pedagógicos, Santiago, v. 35, n. 2, p. 173-188, 2009.

QUINTRIQUEO, S.; TORRES, H. Construcción de Conocimiento Mapuche y su relación con el Conocimiento Escolar. Estudios pedagógicos, Santiago, v. 39, n. 1, p. 199-216. 2013.

ROTHER, T. Conflicto intercultural y educación en Chile: Desafíos y problemas de la educación intercultural bilingüe (EIB) para el pueblo mapuche. Revista Austral Ciencias Sociales, Valdivia, n. 9, p. 71-84, 2005.

SALAS, S. S. Etnomatemática y multiculturalidad en la educación básica en Chile. El caso de la aritmética mapuche.2014. 80p. Tesis (Máster en Didáctica de la Matemática).-Facultad de Educación, 
Universidad de Granada, Granada, España, 2014.

SALAS, S. S.; GODINO, J. D.; OLIVERAS, M. L. Números mapuches en el currículo de la lengua mapuzugun en la educación básica chilena. Revista Latinoamérica de Etnomatemática, San Juan de Pasto, v. 8, n. 2, p. 194-213, 2015.

SKOVSMOSE, O. Hacia una filosofía de la educación matemática crítica. Traducción de VALERO, P. Bogotá: Una Empresa Docente, 1999.

SKOVSMOSE, O. Porvenir y política de los obstáculos de aprendizaje. En: VALERO, P.;

SKOVSMOSE, O., (Coord.). Educación matemática crítica. Una visión sociopolítica del aprendizaje y la enseñanza de las matemáticas. Bogotá: Una Empresa Docente, 2012. p. 131-147.

TREVIÑO, E.; DONOSO, F.; AGUIRRE, E.; FRASER, P.; GODOY, F.; INOSTROZA, D.; CHÁVEZ, B.; PLACE, K.; TAPIA, P.; CASTRO, P. Educación para preservar nuestra diversidad cultural: desafíos de implementación del Sector de Lengua Indígena en Chile. Santiago: Centro de Políticas Comparadas de Educación, Unicef y Mineduc, 2013.

TUBINO, F. El trasfondo epistémico de los conflictos interculturales. Contextualizaciones Latinoamericanas. 2014. Disponible en:

<http://www.contextualizacioneslatinoamericanas.com.mx/index.php>. Acceso en: 13 may. 2015.

VITHAL, R.; SKOVSMOSE, O. The end of innocence: a critique of 'ethnomathematics'. Educational Studies in Mathematics, Holanda, v. 34, n. 2, p. 131-157, 1997.

Submetido em Junho de 2015. Aprovado em Outubro de 2015. 